\title{
Degrees and segments
}

\author{
Roger Schwarzschild \\ Rutgers University
}

\begin{abstract}
I make two related proposals, one about directed scale segments and the other about the nature of degrees. Bale $(2007,2011)$ argued that degrees should be analyzed as sets of individuals and that degree arguments are created in the syntax from relational predicates. Schwarz (2010) showed that Bale's construction runs into problems when the relational predicate is complex, consisting of an LF constituent that contains more than just a gradable adjective. I modify Bale's proposal so that it overcomes Schwarz's objection. But first I propose a semantics for comparatives based on quantification over directed scale segments, triples consisting of two degrees and a measure function. The modification of Bale's proposal depends upon this. Segments are of independent interest as they permit a conjunctive semantics for extended adjectival phrases, the way events do for verb phrases. Potential benefits of 'degree-conjunctivism' are explored.
\end{abstract}

Keywords: Degrees, directed scale segments, comparatives, degree de-re, Navajo, forphrases, than-phrases, conjunction, maximality, monotonicity

\section{Introduction}

It is often said that a gradable predicate such as tall has a degree argument and that constructions such as the comparative or the equative rely on quantification over degrees. I want to consider alternatives to both of these ideas. Section 3 is devoted to the idea that gradable predicates denote relations between possible individuals, an idea discussed in Cresswell 1976 and elsewhere (Hoeksema 1983;

\footnotetext{
* I am indebted to the organizers of SALT 23 at Santa Cruz, participants in the Spring 2013 Semantics Seminar at Rutgers, I-Ta Chris Hsieh, Veneeta Dayal, Pranav Anand, Jane Grimshaw, Renate Raffelsiefen, Jessica Rett, Rajesh Bhatt, Irene Heim, the audience and organizers of the 2012 Linguistics Colloquium at UConn, Magda Kaufmann, Elizabeth Bogal-Allbritten, Chris Kennedy, Jason Merchant, Jonathan Bobaljik, Jon Gajewski, Ezra Keshet, Cecile Meier, Itamar Francez, Sigrid Beck, Alan Bale, Galit Sassoon, Paul Portner, Ken Safir and Todd Snider, the lead editor for the proceedings of SALT 23.

(C) 2013 Schwarzschild
} 
Degrees and segments

Klein 1991; Rullmann 1995: §2.10), most recently in Bale 2011. I summarize Bale's proposals and then offer a modification of his approach that addresses a challenge raised in Schwarz 2010. The modification has to do with how degrees are constructed from relations between individuals. The empirical foci of Bale's proposals are comparatives that include for phrases (taller for a man) and comparatives based on conjunctions (more interesting and dangerous). Section 2 is devoted to the idea that degree constructions rely on quantification over segments of a scale. For example, in a comparative, instead of selecting a degree, $d$, and quantifying over all degrees $d^{\prime}$ that exceed it, we quantify over segments of a scale that begin with some $d$ and end with a higher $d^{\prime}$. This move allows for a conjunctive semantics, in which each part of a comparison (standard, target, greater-than relation) is encoded in its own conjunct. One motivation for this is a comparative construction in Navajo paraphrased by Young and Morgan as "She is pretty, being beyond me", in which the semantics of comparison is all tucked inside a predicative PP, itself buried in an adjunct. A conjunctive semantics also permits an analysis of de-re degree readings of than-phrases (Napoli 1983; Heim 1985; Lechner 2008) using standard tools but without appeal to ellipsis.

The two ideas are logically independent, although both are employed in the solution to Schwarz's challenge and in related examples.

\section{Directed scale segments}

In the comparative in (1) below, Tom is the target of comparison and Susan is the standard of comparison. $\quad-e r$ is a comparative marker and than is a standard marker. I will call the phrase than Susan the standard phrase. The standard phrase has the form of a PP and the entire sentence is a phrasal comparative. This contrasts with a clausal comparative in which the standard marker governs a clause (eg Tom is taller than you think he is).

(1) Tom is taller than Susan.

The formula in (2) below is intended to capture the meaning of (1). Taking TALL to denote the relation that holds between an individual and his height, (2) says that Tom's height exceeds Susan's height.

(2) $\exists d_{\mathrm{t}} \exists d_{\mathrm{s}} \operatorname{TALL}\left(\mathbf{t}, d_{\mathrm{t}}\right) \wedge \operatorname{TALL}\left(\mathbf{s}, d_{\mathrm{s}}\right) \wedge d_{\mathrm{t}}>d_{\mathrm{s}}$

In his commentary on von Stechow 1984, M. J. Cresswell wrote "von Stechow assumes that it is meaningful to say things like $d_{1}>d_{2}$, without saying where $>$ comes from. In particular the question of whether $>$ is an ordering of all degrees, and what kind of an ordering it is, does not seem to me to be really faced." In his own 1976 paper, Cresswell dealt with this issue by taking a degree to be a pair consisting of an ordering and what I will temporarily call a 'measurement'. 
Cresswell would define $d_{\mathrm{t}}$ and $d_{\mathrm{s}}$ as in (3) below. $u_{\mathrm{t}}$ is a measurement, what you ascertain when you measure Tom's height, and $>_{\mathrm{HT}}$ is the standard ordering of heights. In (4), the last conjunct of (2) is spelled out making reference to the coordinates of Cresswellian degrees.

(3) $d_{\mathrm{t}}=\left\langle u_{\mathrm{t}},>_{\mathrm{HT}}\right\rangle, d_{\mathrm{s}}=\left\langle u_{\mathrm{s}},>_{\mathrm{HT}}\right\rangle$

(4) $d_{\mathrm{t}}>d_{\mathrm{s}}$ if and only if $u_{\mathrm{t}}>_{\mathrm{HT}} u_{\mathrm{s}}$

In this paper, I want to explore the consequences of expanding these pairs by adding a measure function, $\mu$, and a second measurement. I want the resulting tuples to pick out segments of a scale and so I restrict them in the following way. The two measurements must be in the set of measurements assigned by the measure function and the ordering must be an ordering of that set. Using $\sigma$ as a variable over these ordered four-tuples, we can write:

$$
\sigma=\langle u, \quad v,\rangle, \mu\rangle
$$

I want to think of the first measurement as the start of the segment and the second as the end. Referring to the segment defined in (5), I introduce special predicates that pick out the first and second coordinates of a scale segment:

$$
\operatorname{START}(\sigma)=u \quad \operatorname{END}(\sigma)=v
$$

A segment that starts with, say, $5 \mathrm{ft}$ and ends with $6 \mathrm{ft}$ is a rising segment. In (7), I introduce a special predicate symbol, $\nearrow$, to say that a segment is rising. In the definition, $>_{\sigma}$ refers to the ordering that is the third coordinate of $\sigma$ and similarly, $\mu_{\sigma}$ will be used to refer to the last coordinate of $\sigma$.

$$
\nearrow(\sigma) \stackrel{\text { def }}{=} \operatorname{END}(\sigma)>_{\sigma} \operatorname{START}(\sigma)
$$

The technical term for a line segment that has a designated start and a designated end is "directed" and so I call my tuples directed scale segments. And I will take the domain of type $d$ to consist of directed scale segments, where, following Hellan 1981 and much recent work, type $d$ is the semantic counterpart of the syntactic label Degree.

We can now give a segmental rendering of the meaning of (1).

(8) $\exists \sigma \nearrow(\sigma) \wedge \operatorname{START}(\sigma)=\mu_{\sigma}(\mathbf{s}) \wedge \operatorname{END}(\sigma)=\mu_{\sigma}(\mathbf{t}) \wedge \mu_{\sigma}=$ HT

(9) 'There is a rising directed scale segment: it starts with Susan's measurement on the scale, it ends with Tom's measurement on the scale, and it is a segment of the height scale.'

Since we are not adopting Cresswell's idea in (3), the term 'degree' is freed up, and I'd like to use it instead of the term 'measurement'. This might cause temporary confusion, but it will facilitate comparison with previous work. So the first two coordinates of a segment are degrees, and (9) should be rephrased as: 
Degrees and segments

(10) 'There is a rising directed scale segment: it starts with Susan's degree on the scale, it ends with Tom's degree on the scale, and it is a segment of the height scale.'

A potential advantage of a segmental semantics lies in the kinds of meanings it allows us to assign to the parts of the comparative clause. To illustrate this potential, let's consider a possible compositional semantics for (1). The structure in (11) below is inspired by the idea that the formula in (8) looks like a Davidsonian event semantic meaning with the segment playing the role of an event variable. The structure is meant to capture the stage before the subject moves to Spec,TP. Below T, I've assumed existential closure of the type $d$ variable, following Rett 2008. I've adopted the idea of an extended AP headed by Deg $^{\circ}$ (see Matushansky 2013 and references therein), with the individual subject of the adjective introduced by a "little deg" (Bhatt and Pancheva use little a). I treat End and Start like thematic role names. End is severed from the adjective like eventive Agent (Kratzer 1996) and Start is notionally like a Source role.

(11) Provisional Syntax and Semantics for phrasal comparative



$$
\begin{aligned}
& \llbracket \text { er } \rrbracket=\lambda \sigma . \nearrow(\sigma) \\
& \llbracket \text { tall }=\lambda \sigma . \mu_{\sigma}=\mathrm{HT} \\
& \llbracket \text { than } \rrbracket=\lambda x \cdot \lambda \sigma .\left(\operatorname{START}(\sigma)=\mu_{\sigma}(x)\right) \\
& \llbracket \operatorname{DegP} \rrbracket=\lambda \sigma . \nearrow(\sigma) \wedge\left(\mu_{\sigma}=\mathrm{HT}\right) \wedge \operatorname{START}(\sigma)=\mu_{\sigma}(\mathbf{s}) \\
& \llbracket \operatorname{End} \rrbracket=\lambda \Sigma . \lambda x . \lambda \sigma .\left(\operatorname{END}(\sigma)=\mu_{\sigma}(x) \wedge \Sigma(\sigma)\right) \\
& \llbracket \operatorname{deg}^{\prime} \rrbracket=\lambda x \lambda \sigma .\left(\operatorname{END}(\sigma)=\mu_{\sigma}(x)\right) \wedge \nearrow(\sigma) \wedge\left(\mu_{\sigma}=\mathrm{HT}\right) \wedge\left(\operatorname{START}(\sigma)=\mu_{\sigma}(\mathbf{s})\right) \\
& \llbracket \operatorname{degP} \rrbracket=\lambda \sigma .\left(\operatorname{END}(\sigma)=\mu_{\sigma}(\mathbf{t})\right) \wedge \nearrow(\sigma) \wedge\left(\mu_{\sigma}=\mathrm{HT}\right) \wedge\left(\operatorname{START}(\sigma)=\mu_{\sigma}(\mathbf{s})\right)
\end{aligned}
$$

The information concerning the kind of scale being described gets introduced with the adjective, but gets passed up and is still present at the $\operatorname{degP}$ level. It is 
encoded in the segments. We'll see that this has favorable consequences for dealing with the properties of comparatives containing for phrases, discussed in Bale 2011. Turning to standard phrases, on the proposed semantics they have two important properties. Like Davidsonian event adverbs, they are one-place predicates and they combine conjunctively. So as far as the semantics is concerned, they are free to combine at any point below the existential closure. Another feature of standard phrases in this setup is that their meaning encodes a measurement of the standard and only of the standard. The two degrees, that of the target and that of the standard, are described in separate expressions. In the next two subsections I present analyses of empirical phenomena that capitalize on these features.

\subsection{Subordinated Standard Phrases (Bogal-Allbritten 2013)}

Bogal-Allbritten 2013 offers a detailed analysis of a comparative construction in Navajo in which the standard phrase is subordinated relative to the rest of the clause. One of her examples is repeated in (12) below, with the standard phrase within square brackets:

$$
\begin{array}{llll}
\text { Shideezhí } & \text { [ shi-lááh } & \text { 'át'ée = go ] } & \text { nizhóní. } \\
\text { my.little.sister } & \text { 1SG.OBJ-beyond } & \text { 3SUBJ.be =SUB } & \text { 3SUBJ.pretty.AA }
\end{array}
$$

'My little sister is prettier than me.'

The bracketed phrase is headed by the clitic $=g o$ which is also used to mark expressions with when-clause and if-clause interpretations (Schauber 1979). This clitic is sometimes referred to as an adverbializer. Bogal-Allbritten also offers syntactic evidence that the bracketed expression is a modifying adverbial. Unlike arguments, the bracketed expression can be moved to the left of the subject. And the bracketed phrase can be omitted altogether preserving grammaticality. The result is glossed as 'my little sister is pretty'.

'át'ée is a copula and shi-lááh is a postpositional phrase that can be used to mean 'beyond me' in the spatial sense. I will assume here that the PP is type $\langle\mathrm{d}, \mathrm{t}\rangle$ which means we have a predicational copula, ${ }^{1}$ type $\langle\langle\mathrm{d}, \mathrm{t}\rangle,\langle\mathrm{d}, \mathrm{t}\rangle\rangle$. Young and Morgan (1987) use paraphrase to give a sense of the meaning of comparatives like those in (12). Their paraphrase for (12) would be "My little sister is pretty, being beyond me" which nicely captures both the predicative nature of the bracketed phrase and its adjunct status. There is one more fact about (12) that will inform our analysis. The postposition -lááh clearly introduces the standard of

\footnotetext{
${ }^{1}$ If we were to assume that PPs are quantificational, then the copula could be taken to include a type shift. See Schwarzschild 2012 for discussion of this point and its relevance to other kinds of comparatives in Navajo.
} 
Degrees and segments

comparison, but it also marks the clause as a comparative of superiority. Replacing -lááh 'beyond' with =gi 'at' leads to an equative meaning, as BogalAllbritten shows, and replacement with -'oh produces a less-than comparative.

Thinking segmentally, -lááh lexicalizes both $\nearrow$ and START predications. The standard phrase has the meaning in (13) below:

$$
\llbracket \text { shi-lááh 'át'ée }=\mathbf{g o} \rrbracket=\lambda \sigma . \quad \nearrow(\sigma) \wedge \operatorname{START}(\sigma)=\mu_{\sigma}(\mathbf{m e})
$$

Assuming something along the lines of our tree above in (11) minus the comparative marker, we get the meaning in (14) which reduces to (15).

$$
\begin{aligned}
& \exists \sigma\left(\operatorname{END}(\sigma)=\mu_{\sigma}(\text { sistr })\right) \wedge \mu_{\sigma}=\operatorname{BEAUTY} \wedge \\
& {\left[\lambda \sigma^{\prime} . \nearrow\left(\sigma^{\prime}\right) \wedge \operatorname{START}\left(\sigma^{\prime}\right)=\mu_{\sigma^{\prime}}(\mathbf{m e})\right](\sigma)} \\
& \exists \sigma\left(\operatorname{END}(\sigma)=\mu_{\sigma}(\text { sistr })\right) \wedge \mu_{\sigma}=\operatorname{BEAUTY} \wedge \nearrow(\sigma) \wedge \operatorname{START}(\sigma)=\mu_{\sigma}(\mathbf{m e})
\end{aligned}
$$

If the standard phrase is omitted from (12), the interpretation we get is the top part of (14), which only says that my sister has some measure of beauty. Evaluativity may need to be added via a Rettian EVAL predicate or by restricting the domain of existential closure (see Bogal-Allbritten 2013 and Schwarzschild 2012 for discussion).

On the just proposed analysis, -lááh is able to encode both $\nearrow$ and START in an independent, subordinated modifier because our domain of quantification consists of segments and a segment is capable of possessing a start point and of having an orientation. The identity of the measurement function on which $\nearrow$ is defined need not be revealed in the adjunct, because it is described in the main predication and the information is carried along in the segment. Apparently, languages can choose to apportion these pieces of information in the opposite way with $\nearrow$ and START in the main predication and the measure function in the modifier, as in Andersen's 1982: 233 (1) example of a 'verbal construction':

(16) John surpasses his friends in skill.

In (16), the main verb encodes $\nearrow$ and the roles END and START are associated with subject and object positions. The identity of the measure function follows in the modifier. ${ }^{2}$

According to what we claimed here in (13), and in the previous section in (11), Navajo -lááh encodes $\nearrow$ but English than does not. The claim about English isn't completely true to the facts, for even if $\nearrow$ is encoded in $-e r$, the presence of than is a sign that a rising segment is being described. ${ }^{3}$ We can capture this state

\footnotetext{
${ }^{2}$ I'm grateful to Philippe Schlenker for pointing out the relevance of a segment analysis to 'exceed comparatives', to use Stassen's (1985) term.

${ }^{3}$ Let's assume that less is a spellout of little + -er (Bobaljik 2012 §7.2; Heim 2008 and references therein).
} 
of affairs by adding a presupposition (enclosed between a colon and a dot) to the meaning of than as in (17) or (18).

$$
\begin{aligned}
& \llbracket \text { than } \rrbracket=\lambda x . \lambda \sigma: \nearrow(\sigma) . \operatorname{START}(\sigma)=\mu_{\sigma}(x) \\
& \llbracket \text { than } \rrbracket=\lambda x . \lambda \Sigma: \forall \sigma(\sigma \in \Sigma \rightarrow \nearrow(\sigma)) . \lambda \sigma . \operatorname{START}(\sigma)=\mu_{\sigma}(x) \wedge \Sigma(\sigma)
\end{aligned}
$$

With (18), function application is used to interpret the merging of the standard phrase with the rest of the clause. The motivation for (18) is that we may want to treat equative as as describing segments that are either rising or flat. In that case, with (17) we run the risk of allowing *as tall than, depending on how presupposition projection works in intersective modification structures. On the other hand, this potential disadvantage of (17) for English would be an advantage for languages in which the standard marker for equatives and comparatives is the same (Andersen 1982: 236, von Stechow 1984: 8). The presuppositional proposal in $(17) /(18)$ might be a good alternative to the somewhat problematic approaches to the -er than dependency in terms of selection or agreement (Alrenga, Kennedy, and Merchant 2012: 39, Bhatt and Pancheva 2004: 3-4, Grosu and Horvath 2006: 459). This proposal also offers a different perspective on the point of attachment of than clauses relative to the position of -er, an issue discussed at length in the above referenced works. Assuming (18), in order to satisfy the presupposition, than phrases must be merged after -er, and for type-reasons, they must be merged before existential closure.

In this subsection, we looked at free-adjunct standard-phrases in Navajo, the -er than dependency and exceed comparatives. In each case, I've highlighted the potential advantages of a semantics that runs on segments, but without seriously comparing it with the alternatives. For the Navajo data, Bogal-Allbritten addresses the lack of a measure function description in the standard phrase by appeal to ellipsis. In Navajo, there is a kind of subcomparative in which the object of the postposition is a nominalized clause. Based on this, Bogal-Allbritten posits an underlying clause inside the adjunct in (12) above. In that case, the second, elided, occurrence of the adjective (re)identifies the measure function. Ellipsis will figure in a similar way in the following section on de-re readings.

\subsection{De-re standard phrases}

In the proposed theory, the degree of the standard is encoded in its own predicate, the standard phrase, to the exclusion of the degree of the target of comparison. This means that if a comparative is found in an intensional context and the standard phrase is read de-re while the rest of the comparative is read de-dicto, we'll in effect be comparing degrees reached in different possible worlds. To see what this looks like, consider the counterfactual in (19) below: 
Degrees and segments

(19) If they had hired Holly, at least she would have been nicer to the staff than the current manager.

(19) compares how nice Holly would have been to how nice the current manager actually is. The modal statement below also has a salient de-re reading of the standard phrase:

(20) According to the code, the new bench has to be wider than that old bench over there.

(20) would be felicitous in a context where the production of a new bench is being discussed and where the speaker wants to use a nearby object to pick out a width. Crucially, the code in question makes no reference to the relative widths of the benches: if a too narrow new bench is produced, shortening the old one won't help. (20) compares how wide the new bench is in code-sanctioned worlds to how wide the old bench is in the actual world.

In both (19) and (20), the DP object of than is read de-re, but that is not sufficient to produce the interpretation we're after. As Heim (1985: §3.4) observes, the degree-predication itself needs to be transparent. To capture this reading in (20), we'll need a meaning for wide and we'll need to update some of the meanings given in (11) above (I'm ignoring the presupposition of than introduced in (17)-(18)).

ide $=\lambda \sigma \cdot \mu_{\sigma}=\mathrm{WIDTH}$

(22) WIDTH is a function whose domain includes all and only pairs of a world $w$ and an object $x$ in $w$ that has a width.

For any $\langle x, w\rangle$ in its domain, $\operatorname{WIDTH}(w)(x)$ is $x$ 's width in $w$.

$$
\begin{aligned}
& \llbracket \text { than } \rrbracket=\lambda w \lambda x \lambda \sigma .\left(\operatorname{START}(\sigma)=\mu_{\sigma}(w)(x)\right) \\
& \llbracket \operatorname{End} \rrbracket=\lambda w \lambda \Sigma \lambda x \lambda \sigma .\left(\operatorname{END}(\sigma)=\mu_{\sigma}(w)(x) \wedge \Sigma(\sigma)\right)
\end{aligned}
$$

And now assuming a syntax as in (11) above, we can capture the intended interpretation of (20) in a semantics with object level world variables as follows: ${ }^{4}$

(25) $\operatorname{has}_{w} \lambda w^{\prime}$ (to-be $\exists \sigma$ the new-bench $w^{\prime} \operatorname{End}_{w^{\prime}}$ wider than ${ }_{w}$ the old-bench ${ }_{w}$ )

$$
\begin{aligned}
\forall w^{\prime}\left(\operatorname{ACC}\left(w^{\prime}, w\right) \rightarrow \exists \sigma(\operatorname{END}(\sigma)=\right. & \left.\mu_{\sigma}(\text { new })\left(w^{\prime}\right)\right) \wedge \nearrow(\sigma) \wedge\left(\mu_{\sigma}=\mathrm{WIDTH}\right) \wedge \\
& \left.\left(\operatorname{START}(\sigma)=\mu_{\sigma}(w)(\mathbf{o l d})\right)\right)
\end{aligned}
$$

In (25), the standard phrase is a predicate that is read relative to a different world of evaluation than its neighbors. Predicates can also display temporal independence from their neighbors (Cooper 1978: 153; Enç 1986; Musan 1999;

\footnotetext{
${ }^{4}$ In the SALT talk, I treated this example in terms of Schwager's (2011) analysis of 'Reading 3' which is based on Cresswell and von Stechow's higher order de-re. I took the reported property to be the property of being a width segment that starts with the width of the old bench.
} 
Kusumoto 2005; Tonhauser 2007a,b; Keshet 2010) and standard phrases are no exception. As McConnell-Ginet (1973: 159-160) observed, (27) below is naturally read as comparing our heights, measured now, to our ancestors' heights, measured in the past.

(27) We are taller than our ancestors.

Just as standard-phrase PPs have transparent readings, so do temporal and spatial PPs, as in the following examples:

(28) The midterm exam has $\lambda w^{\prime}$ to take place before ${ }_{w}$ our next potluck, according to University rules.

(29) You see Beal over there, standing against the wall? The painting needs $\lambda w^{\prime}$ to be about 3 inches above ${ }_{w}$ his head.

(28) locates the exam relative to the time of the potluck, but of course University rules don't refer to potluck time, and a postponement of the potluck will not allow for a later midterm. In (29), the location of the painting in the need fulfilling world-times is coordinated with the location of Beal's head in the actual world at the moment of speech.

The analysis in (25) relies on the fact that the meaning of standard marking than includes the application of a measure function. As in the previous subsection, there is an alternative analysis that relies on ellipsis. Lechner (2008: §7.2.2) maintains that phrasal comparatives in English result from ellipsis in a clausal complement and he attributes de-re readings of standard phrases to contra-world-indexing the elided material and its antecedent. On this account, the source for (20) above could be:

(30) The new bench has ${ }_{w} \lambda w^{\prime}$ to be wider $w^{\prime}$ than that old bench is wide $w^{\text {. }}$

If we ignore Heim's (1985: §3.4) suggestion that contra-indexing blocks ellipsis, then (30) presents a real alternative to the segmental analysis, for English. However, Bhatt and Takahashi argue that standard phrases in Hindi are never underlyingly clausal and the Hindi example below (Veneeta Dayal, pc) has a dere readable standard phrase, just like (20):

naye bench-ko is bench-se aaT $\mathrm{T}^{\mathrm{h}}$ inch cauRaa honaa caahiye
new bench-DAT this bench-SE 8
'The new bench has to be 8 inches wider than this bench'

It remains to be seen how the segmental analysis or the ellipsis analysis could explain the contrasts below, (32) from Heim 1985 and (33) from McConnellGinet 1973. In both cases the phrasal comparative in (a) lacks a sensible, de-re reading, present in its clausal counterpart in (b). 
Degrees and segments

(32) a. If your sister hadn't been born, they would have spoiled you even more than her.

b. If your sister hadn't been born, they would have spoiled you even more than they spoiled her.

(33) a. Sydney is taller than Plato.

b. Sydney is taller than Plato was.

In this subsection, we've covered so-called de-re degree readings of phrasal comparatives. These readings are predicted by the segmental analysis sketched in (11) in conjunction with standard analyses of de-re read predicates. ${ }^{5}$ But again, at least in some cases, there are clausal ellipsis alternatives. And even within nonellipsis accounts, it is possible to encode measurement in the meaning of than without having a conjunctive, segmental, semantics. ${ }^{6}$ Faller (2000) and following her Winter (2005) offer analyses along these lines. One problem that Faller acknowledges (footnote 8) and that carries over to the present account has to do with "complicated sentences" like the producer wants the film to be more violent than the director. As pointed out by a reviewer, Faller's analysis cannot capture a reading of that sentence where the than-phrase is synonymous with than the director wants it to be. This issue will be addressed in section $3 .^{7}$

\section{Constructing degrees}

In this section we'll be working towards a framework in which degrees are not primitive, but rather are defined in terms of sets of possible individuals. Subsection 1 gives background and motivation. Subsection 2 offers a proposal for defining degrees and then using them to create directed scale segments. Subsection 3 returns to the issues raised in subsection 1 .

\subsection{Bale, Kennedy \& Schwarz}

In the example in (34), we have a conjunction of adjectives under a comparative marker.

(34) The rattlesnake is more aggressive and poisonous than the copperhead.

\footnotetext{
${ }^{5}$ The implementation in (25) does however violate Keshet's 2010: 388 Intersective Predicate Generalization which bans contra-world-indexing of "two predicates interpreted intersectively". ${ }^{6}$ Yet another possibility is double world indexing of -er, along the lines of the treatment of too in Nissenbaum and Schwarz 2008: $§ 5.3$.

${ }^{7}$ Some years ago, I tried a degree analysis with standard phrases that worked like Faller's. I am grateful to Irene Heim for making clear to me then the problem posed by these complicated sentences. I tried a somewhat ad hoc solution at SALT 22 .
} 
Bale (2007) observed that examples of this type are problematic if one holds that (a) gradable adjectives have degree arguments, distinct ones for different kinds of scales, and (b) predicate conjunction is interpreted as generalized intersection. If degrees of aggression are different from degrees of toxicity, the intersection will be empty. Bale proposed to solve this problem by introducing degree arguments with the degree morphology. Here's a rough idea of how that works. We start with an $\langle e,\langle e, t\rangle\rangle$ meaning like in (35):

$$
\text { 【aggressive }=\lambda x . \lambda y . x \text { is at least as aggressive as } y
$$

Using this meaning we define metalanguage relations equally aggressive and $\underline{\text { more aggressive than. }}$

$$
x \text { is equally as aggressive as } y \text { iff }
$$

(37) $x$ is more aggressive than $y$ iff

$$
\llbracket \text { aggressive } \rrbracket(x)(y)=1 \text { \& \aggressive } \rrbracket(y)(x)=0
$$

Now we can identify $x$ 's degree of aggression with the set of individuals who are equally as aggressive as $x$. This set is called $x$ 's equivalence class. Next we define a relation that orders degrees of aggression: $d_{1}>d_{2}$ just in case any individual in the class/degree $d_{1}$ is more aggressive than any individual in the class/degree $d_{2}$. Finally, we define an individual-degree relation: $\lambda x \lambda d . x \in d$. Further details can be found in Bale 2007, 2011. The crucial point here is that the meaning in (35) relates individuals and so the conjunction in (34) can happen before degrees are introduced. And then when more is added in (34), a degree relation is created starting with the $\langle e,\langle e, t\rangle\rangle$ meaning of aggressive and poisonous. $^{8}$

In Bale 2011 we find a different kind of argument for constructing degrees out of individual relations. The argument is based on examples like (38) below:

(38) Esme is taller for a woman than Seymour is for a man.

Bale's reasoning begins with Kennedy (2007) who argued that for phrases are domain restrictors on functions denoted by gradable predicates. tall for a man denotes a function that is defined only for men. As Kennedy argues, the impression that for phrases pick out comparison classes results from the fact that a norm for comparison is calculated on the restricted function. When we inspect the range of possible heights assigned, to arrive at a standard, we only look at heights of men because only they are assigned heights by this function. But if a for phrase merely restricts a degree function, as Kennedy has it, we predict (38)

\footnotetext{
${ }^{8}$ Faller (1999: 168) foresees exactly this type of solution to the conjunction problem.
} 
Degrees and segments

above to be synonymous with (39), assuming Esme is woman and Seymour is a man:

(39) Esme is taller than Seymour is.

In fact, (38) and (39) are not synonymous. It could be that (38) is true and (39) is false. (38) induces what Bale calls 'indirect comparison' which 'involves comparing the relative position of Esme in an ordering of women based on height to the relative position of Seymour in an ordering of men based on height." This is the same kind of interpretation that he observes in (40).

(40) Marie Curie is more intelligent than Marilyn Monroe is beautiful.

Here the relative position on an intelligence scale is compared with the relative position on a beauty scale. The indirect interpretation is triggered in (40) by the fact that we compare different kinds of degrees. Likewise, Bale takes the presence of indirect comparison in (38) to be evidence that the degrees satisfying tall for a woman and tall for a man are distinct. He derives this result by allowing the for phrases to restrict type $\langle e,\langle e, t\rangle\rangle$ expressions giving us meanings like in (41)-(42):

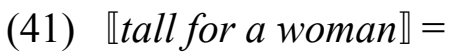

$\lambda x: x$ is a woman. $\lambda y: y$ is a woman. $x$ is at least as tall as $y$.

(42) $\llbracket$ tall for a man $\rrbracket=$

$\lambda x: x$ is a man. $\lambda y: y$ is a man. $x$ is at least as tall as $y$.

Degrees are constructed from (41) and (42). But now the equivalence classes will be different - consisting entirely of women in the one case and entirely of men in the other (recall (36)). Since these equivalence classes are the degrees, we end up with distinct degrees as in (40).

Schwarz (2011) points out that Bale's construction works well when the for phrase modifies an adjective, but it breaks down in more complex cases. Schwarz argues that in (43),

(43) Mia has an expensive hat [for a 3-year old].

the for phrase restricts a derived degree function, one that is denoted by a constituent formed at LF. The restricted function assigns to a 3 year old the price of the hat they have:

(44) $\lambda x: x$ is 3 yrs old. $\lambda d$. $x$ has a $d$-expensive hat.

There are two facts about (43) that implicate the meaning in (44). First, it is Mia who is presupposed to be a 3 year old, not the understood subject of expensive, which is a hat. Secondly, the norm of comparison is the expected price of a hat that a 3 year old might have. That's because we consider the range of values, hat 
prices, that the degree function in (44) assigns to elements in its domain, 3 year olds. But now consider what would happen if we tried to arrive at (44) on Bale's approach. We'd presumably form degrees starting with the type $\langle e,\langle e, t\rangle\rangle$ function in (45):

(45) $\lambda x: x$ is 3yrs old. $\lambda y: y$ is 3yrs old. $x$ has a hat that is at least as expensive as $y$.

By the construction in (35)-(36) above, if Tom is in the same equivalence class as Mia, then it has to be that $\llbracket(45) \rrbracket(\mathbf{m i a})($ tom $)=\llbracket(45) \rrbracket($ tom $)(\mathbf{m i a})=1$. But $\llbracket(45) \rrbracket($ tom $)(\mathbf{m i a})=1$ says that Tom has a hat that is as expensive as Mia is. In general, assuming Mia does not have a price, there will be no equivalence class, hence no degree, to pair her with. More technically, Bale's construction relies on adjectives denoting relations that are reflexive, but reflexivity is not guaranteed for the meanings of complex constituents containing adjectives.

As Schwarz shows, this particular example, (43), could be handled by the original Kennedy method where a for phrase restricts a measure function, one that is denoted by a constituent formed at LF. But if we give up on Bale, we lose our explanation for the Esme-Seymour example, (38). And, Schwarz argues, it will not do to limit Bale's method to simple cases, since for induced indirect interpretations arise with derived degree functions as well:

(46) Mia has a more expensive hat [for a 3-year old] than Sam does [for a 9-year old].

My aim in the remainder of this section is to address Schwarz's objection by modifying Bale's construction of degree predicates.

\subsection{Constructing degrees and directed scale segments}

Bale's construction of degrees follows Cresswell, who was worried about "the metaphysical reality of degrees of comparison". As we have just seen, the Achilles heel of this construction is reflexivity, so the plan is to modify the construction in some way that avoids it. In building up to his proposal, Cresswell (1976: 281) writes: "We take as basic data that we can and do make comparisons, i.e., that comparative sentences can be true or false; further, that we have the ability to make counterfactual comparisons. That is to say, our competence in this area is not limited to judgments involving how things actually are but can encompass judgments about how things might be. This means that we can make other-world and even trans-world comparisons." With this idea in mind, I propose that we take gradable adjectives to relate possible individuals. Following Cresswell, I'll represent a possible entity with a pair of a thing and a world. Ignoring the presupposition of heightedness, the meaning of tall comes out as: 
Degrees and segments

$$
\llbracket \text { tall } \rrbracket=\lambda w \cdot \lambda x \cdot \lambda\left\langle y, w^{\prime}\right\rangle, x^{\prime} \text { s height in } w \text { meets or exceeds } y \text { 's height in } w^{\prime} .
$$

Blurring the distinction between sets and their characteristic functions, according to (47), when applied to a world $w$ and an entity $x$, $\llbracket t a l l \rrbracket$ assigns $x$ a set of possible individuals that are equally tall or less tall than $x$ in $w$. I propose that we identify these sets of possible individuals with degrees. The motivating intuition is that when we name a person's height, we aim to convey where $x$ is situated in a height ordering relative to all other entities, real and imagined.

Once we have degrees, we can proceed to the other elements of a directed scale segment, the ordering and the measure function. If a set of possible individuals is a degree, then the meaning for tall in (47) just is a measure function (compare the definition of WIDTH given in (22)) and proper subset gives us what we need for an ordering:

(48) For any degrees, $u, v: u<v$ IFF $u \subset v$.

Taking (47) to be a height-assigning measure function, according to (48), if all those possible individuals who are as tall or less tall than Jack are also as tall or less tall than Jill, but not vice-versa, then Jack's height is lower than Jill's. Going back to Cresswell's commentary on von Stechow 1984 cited at the beginning of section 2, on the current conception of degrees, the ordering in fact is an ordering of all degrees. And now, since it is the same ordering no matter what, it no longer has to be specified in our segments.

Here's a summary of the revised segmental framework:

Segmental Semantic Framework (Revised)

Degree

the characteristic function of a set of entity- $u, v$ world pairs $(\approx$ set of possible individuals $)$

Measure function

a function that assigns a degree to an entity in a world

Directed scale segment a 3-tuple whose third coordinate is a measure function and whose first two coordinates are degrees in the range of that measure function

(50) Notation

Let $\sigma=\langle u, v, \mu\rangle$ be a directed scale segment, then:

$$
\operatorname{START}(\sigma)=u, \quad \operatorname{END}(\sigma)=v, \quad \nearrow(\sigma) \stackrel{\text { def }}{=} \operatorname{START}(\sigma)<\operatorname{END}(\sigma)
$$


The adjective meaning in (47) relates individuals, so at some point segments need to be introduced. I follow Bale (2007) in including the introduction of $d$-type arguments in the meaning of the comparative marker. I use the label $e \times s$ for the type of possible individuals.

$$
\llbracket-e r \rrbracket=\lambda \mathcal{A}_{<\mathrm{s},<\mathrm{e},<\mathrm{e} \times \mathrm{s}, \downarrow>>>} \lambda \sigma . \nearrow(\sigma) \wedge \mu_{\sigma}=\mathcal{A}
$$

The main changes relative to previous sections are these: segments no longer include an ordering, degrees are analyzed in terms of possible individuals, adjectives denote measure functions (as in Kennedy 1997 and much subsequent work), and finally, the meaning of -er applies to a measure-function meaning and introduces segments. The rest of the analysis in (11) can remain the same.

Let us now look at some of the examples that motivated Bale's construction of degrees in the syntax. In the snake example, (34), the meaning of aggressive and poisonous can be gotten by intersecting the relations denoted by the two adjectives, giving us a new measure function that, when applied to a world $w$ and an entity $x$, assigns a set containing any possible entity that is situated at or below $x$ on the aggression ordering and at or below $x$ on the toxicity ordering. (34) says that the degree of the rattlesnake on this scale is greater than, that is, a proper superset of, that of the copperhead. If, for example, the snakes are equally aggressive and equally toxic, then (34) is correctly predicted to be false, since in that case there are no possible individuals in the copperhead's degree that aren't also in the rattlesnake's. ${ }^{9}$

We turn now to Bale's other argument for constructing degrees in the syntax: comparatives with for phrases in them. Let's assume with Bale that the for phrase is combined before -er, but that it only restricts the $e$-type argument, giving us the meaning in (52).

\section{$\llbracket$ tall for a woman $=$}

$\lambda w . \lambda x: x$ is a woman in $w . \lambda\left\langle y, w^{\prime}\right\rangle . x$ 's height in $w$ meets or exceeds $y$ 's height in $w^{\prime}$.

\footnotetext{
${ }^{9}$ This semantics predicts that (34) is true when the snakes are equally toxic but the rattlesnake is more aggressive. A numerical illustration should make this clear. Suppose the rattlesnake scores a 6 on the aggression scale and a 4 on the toxicity scale. In that case, he scores a $(6,4)$ on the aggression-toxicity scale. And suppose the copperhead scores a $(4,4)$ on that scale. In that case, the copperhead's degree will be a subset of the rattlesnake's, and it will be a proper subset, because, for example, an entity that scores $(5,3)$ is in the rattlesnake's degree but not the copperhead's. I'm not sure what the intuitions are in this case. It is possible that the sentence is infelicitous due to a homogeniety presupposition associated with the conjunction (see Gajewski 2005: §4-5, Szabolcsi and Haddican 2004, Malamud 2012 and references therein). I'm grateful to Galit Sassoon and Paul Portner for discussion of this issue.
} 
Degrees and segments

Let's further assume with Alrenga et al. that in clausal comparatives there is an instance of -er in the main clause and in the comparative clause. In that case, we have the following meanings:

$$
\begin{aligned}
& \llbracket \text { taller for a woman }=\lambda \sigma . \nearrow(\sigma) \wedge \mu_{\sigma}= \\
& \lambda w . \lambda x: x \text { is a woman in } w . \lambda\left\langle y, w^{\prime}\right\rangle . x \text { 's height } \\
& \llbracket \text { taller for a man }=\lambda \sigma . \quad \nearrow(\sigma) \wedge \mu_{\sigma}= \\
& \text { in } w \text { meets or exceeds } y^{\prime} \text { s height in } w^{\prime} \text {. } \\
& \lambda w . \lambda x: x \text { is a man in } w . \lambda\left\langle y, w^{\prime}\right\rangle . x \text { 's height in } \\
& w \text { meets or exceeds } y \text { 's height in } w^{\prime} \text {. }
\end{aligned}
$$

The segments described in (53) and (54) do not have the same measure function. In other words, the $d$ type arguments in the main clause and the comparative clause of (38) are different, just as they are in (40). In both cases the segments described in the main and subordinate clauses are based on different measure functions. Suppose that the main and subordinate clause of (38) and (39) above are interpreted as in our original (11), but without the existential closure. And then suppose the two clausal meanings are combined with the meaning in (55).

$$
\begin{array}{r}
\llbracket \text { than }_{\text {clausal }} \rrbracket=\lambda \Sigma . \lambda \Sigma^{\prime}: \exists \sigma \exists \sigma^{\prime} \sigma \in \Sigma \wedge \sigma^{\prime} \in \Sigma^{\prime} \wedge \mu_{\sigma}=\mu_{\sigma^{\prime} .} \exists \sigma \exists \sigma^{\prime}(\sigma \in \Sigma \wedge \\
\left.\sigma^{\prime} \in \Sigma^{\prime} \wedge \operatorname{END}(\sigma)=\operatorname{START}\left(\sigma^{\prime}\right)\right)
\end{array}
$$

(39) will be felicitous and it will be true if Esme is taller than Seymour. In that case, as (55) requires, there will be a rising segment $\sigma$ that ends with Seymour's height and a rising segment $\sigma^{\prime}$ that ends with Esme's height such that the start of $\sigma^{\prime}$ is the end of $\sigma$. (38), Esme is taller for a woman than Seymour is for a man, will suffer presupposition failure, as desired. This sketch is not meant to be a serious analysis of clausal comparatives. ${ }^{10}$ My aim is to demonstrate that within a segmental semantics, it is possible to capture Bale's essential idea: even though for phrases are merely domain-restrictors, their use affects the nature of constructed $d$-type arguments and thus only an indirect interpretation is possible in comparatives formed over for phrase restricted adjectives.

Finally, we come to Schwarz's example:

$$
\text { Mia has an expensive hat [for a 3-year old]. }
$$

\footnotetext{
${ }^{10}$ See Beck 2012 for an analysis of a rich array of facts to do with modals and quantifiers in comparative clauses. Although I have not said how to deal with those facts, it is worth noting that conjoined adjectives can be used to form clausal comparatives:

(i) The rattlesnake is more aggressive and toxic than at least one of the copperheads was. This type of example helps to rule out a conjunction reduction source for adjectives conjoined under more. (i) is not equivalent to:

(ii) The rattlesnake is more aggressive than at least one of the copperheads was and is more toxic than at least one of the copperheads was.
} 
Recall that a key feature of this example is that the restriction to 3 year olds applies to the subject of the main verb, so assuming Kennedy's analysis of for phrases, Schwarz deduces the presence of a type $\langle d,\langle e, t\rangle\rangle$ predicate formed at LF that includes the whole VP. Schwarz attributes the creation of the derived predicate to LF movement of the operator POS, generated adjacent to the adjective. I will adapt Schwarz's proposal to the current context. To follow the derivational steps Schwarz assumes, we need to reorder the arguments of adjectives, as in (57).

$$
\begin{gathered}
\llbracket \text { expensive }=\lambda w . \lambda\left\langle y, w^{\prime}\right\rangle . \lambda x . x \text { 's price in } w \text { meets or exceeds } y \text { 's price in } \\
w^{\prime} .
\end{gathered}
$$

This meaning still relates an individual and a world to a set of possible individuals, but since the type is changed, instead of saying that the measure function coordinate is equal to the meaning given by the gradable relation, we'll have to say it is congruent to it:

$$
\mu \approx \mathcal{B}_{<\mathrm{s},<\mathrm{e} \times \mathrm{s},<\mathrm{e}, \mathrm{t}>>>} \stackrel{\text { def }}{=} \mu=\lambda w . \lambda x . \lambda\left\langle y, w^{\prime}\right\rangle \cdot \mathcal{B}(w)\left(\left\langle y, w^{\prime}\right\rangle\right)(x)
$$

The set of possible individuals assigned to any $x$ in $w$ by (57) is an indicator of where $x$ is on an ordering based on monetary value and I will refer to such a set as 'a price'.

In steps (59)-(61), the sentence is generated with POS adjacent to the adjective, the subject Mia is raised, creating a $\lambda$-abstract, and then POS raises to the edge of that newly created abstract. This type of derivation is discussed in Barker 2007: $\S 6$ who called it 'parasitic scope taking'. It was exploited in Heim 1990 for phrasal comparatives and is now widespread (see Dotlačil 2010: 61 and references therein). The world-binder $\lambda w$ is introduced in (62) because POS takes an intensional argument.

(59) Mia has a POS expensive hat.

(60) Mia $\lambda x x$ has a POS expensive hat.

(61) Mia POS $\lambda\left\langle y, w^{\prime}\right\rangle \lambda x x$ has a $\left\langle y, w^{\prime}\right\rangle$-expensive hat.

(62) Mia POS $\lambda w \lambda\left\langle y, w^{\prime}\right\rangle \lambda x x$ has a $\left\langle y, w^{\prime}\right\rangle$-expensive hat.

At this point, the adjunct for a 3 year old is merged countercyclically with the sister of POS:

(63) Mia POS [[ $\lambda w \lambda\left\langle y, w^{\prime}\right\rangle \lambda x x$ has a $\left\langle y, w^{\prime}\right\rangle$ expensive hat.] [for a 3 year old]]

The meanings for the for phrase and for POS are in (64) and (65) and the resulting meaning for the sentence is in (66). The world arguments in (66) originate from a world-indexed structure, as in section 2.2. 
Degrees and segments

(64) $\llbracket$ for a 3 year old $\rrbracket=\lambda \mathcal{B} . \lambda w . \lambda\left\langle y, w^{\prime}\right\rangle . \lambda x$ : $\mathrm{x}$ is 3 yrs old in $w . \mathcal{B}$ $(w)\left(\left\langle y, w^{\prime}\right\rangle\right)(x)$

$$
\begin{array}{r}
\llbracket \mathrm{POS} \rrbracket=\lambda \mathcal{B} . \lambda w . \lambda x . \quad \exists \sigma \quad \nearrow(\sigma) \wedge \operatorname{END}(\sigma)=\mu_{\sigma}(w)(x) \wedge \operatorname{sTART}(\sigma)= \\
\operatorname{NORM}\left(\mu_{\sigma}\right)(w) \wedge \mu_{\sigma} \approx \mathcal{B}
\end{array}
$$

(66) $\exists \sigma \nearrow(\sigma) \wedge \operatorname{END}(\sigma)=\mu_{\sigma}(w)($ mia $) \wedge \operatorname{START}(\sigma)=\operatorname{NORM}\left(\mu_{\sigma}\right)(w) \wedge$

$\mu_{\sigma}=\lambda w . \lambda x: x$ is 3 yrs old in $w . \lambda\left\langle y, w^{\prime}\right\rangle . x$ has a hat whose price in $w$ exceeds $y$ 's price in $w^{\prime}$.

$\mu_{\sigma}(w)$ (mia) is only defined if Mia is a 3 year old in $w$. And the norm will be calculated on a function that only applies to 3 year olds and returns hat prices. (66) requires Mia' hat price to be above the norm. We have succeeded then in implementing Schwarz's proposal in a Bale-friendly framework, where gradable adjectives denote relations between individuals.

\subsection{Phrasal comparatives: direct analysis}

In certain respects, the Schwarz-Bale interaction recapitulates an earlier one between Heim and Hoeksema. Hoeksema (1983) associated adjectives with a 'primitive grading relation' of type $\langle e,\langle e, t\rangle\rangle$. His semantics for phrasal comparatives begins by using that relation to assign a meaning to the comparative form of the adjective. Heim (1990) investigated the matter in detail and concluded "...that comparative morpheme affixation not only resists a lexical treatment, but [that] the comparative suffix must even be assumed to move in the derivation of Logical Form (LF) from S-Structure (SS)." Heim 2001 covers a wealth of additional evidence for this view. Since Schwarz's own analysis was informed by Heim's discovery, it is no surprise that a derivation similar to (59)(61) above, can be used in (67) below, with more taking the place of POS.

(67) Mia has a more expensive hat than Zelda.

(68) Mia has a more expensive hat.

(69) Mia $\lambda x x$ has a more expensive hat.

(70) Mia more $\lambda\left\langle y, w^{\prime}\right\rangle \lambda x x$ has a $\left\langle y, w^{\prime}\right\rangle$ expensive hat.

(71) Mia more $\lambda w \lambda\left\langle y, w^{\prime}\right\rangle \lambda x x$ has a $\left\langle y, w^{\prime}\right\rangle$ expensive hat.

(72) Mia [more $\lambda w \lambda\left\langle y, w^{\prime}\right\rangle \lambda x x$ has a $\left\langle y, w^{\prime}\right\rangle$ expensive hat] [than Zelda].

(73) Mia END [more $\lambda w \lambda\left\langle y, w^{\prime}\right\rangle \lambda x x$ has a $\left\langle y, w^{\prime}\right\rangle$ expensive hat] [than Zelda].

(74) $\exists \sigma$ Mia END [more $\lambda w . \lambda\left\langle y, w^{\prime}\right\rangle . \lambda x x$ has a $\left\langle y, w^{\prime}\right\rangle$ expensive hat] [than Zelda].

To keep in sync with section 2, I continue to assume a meaning for more like in (51) in the previous subsection. For this reason, I've inserted an End head in (73). 
This means that Mia is assigned two thematic roles, one in the VP and one in the degP, making this a kind of control via movement structure (Boeckx and Hornstein 2004). ${ }^{11}$ An obvious alternative would be to complicate the meaning of more so that it introduces the target of comparison. The meaning in (74), like (66), asserts the existence of a rising segment that ends with Mia's hat-price, but this time the segment starts with Zelda's hat-price.

Assuming a similar kind of derivation via more movement in Schwarz's (75) below, the segments described in the main and subordinate clauses will have different measure function coordinates, as in Bale's original example (38).

(75) Mia has a more expensive hat [for a 3-year old] than Sam does [for a 9-year old].

This completes our discussion of Schwarz's challenge to Bale. I'd like to return to (76) below in a wider context of degree semantics. On the current proposal, the interpretation of (76) makes use of the measure function in (77):

(76) Mia has a more expensive hat than Zelda.

(77) $\lambda w . \lambda x . \lambda\left\langle y, w^{\prime}\right\rangle . x$ has a $\left\langle y, w^{\prime}\right\rangle$-expensive hat

If Zelda has several hats, (77) will assign her the price of the most expensive one: the set of all possible entities whose price is less than a hat Zelda has. This means that our semantics for (76) requires Mia to have some hat that is more expensive than any Zelda has. An analysis of Heim's which uses a maximality operator in the meaning of -er makes the same prediction, which Heim 1990: 40 finds questionable (see also Sharvit and Stateva 2002: §6.2)

The maximality operator was introduced into degree semantics by von Stechow (1984), who was motivated in part by examples with possibility modals in than-clauses. von Stechow did not consider necessity modals. Heim (2001) later showed that incorporating necessity modals in a system like von Stechow's requires degree relations to be monotonic, in a sense to be defined shortly.

\footnotetext{
${ }^{11}$ The control-as-movement description applies more aptly in a derivation with traceless movement instead of parasitic scope taking:

(i) Mia has a more expensive hat.

(ii) Mia $\lambda x x$ has a more expensive hat.

(iii) more $\lambda\left\langle y, w^{\prime}\right\rangle$ Mia $\lambda x x$ has a $\left\langle y, w^{\prime}\right\rangle$-expensive hat.

(iv) more $\lambda w \lambda\left\langle y, w^{\prime}\right\rangle$ Mia $\lambda x x$ has a $\left\langle y, w^{\prime}\right\rangle$-expensive hat.

(v) End [more $\lambda w \lambda\left\langle y, w^{\prime}\right\rangle$ Mia $\lambda x x$ has a $\left\langle y, w^{\prime}\right\rangle$-expensive hat] [than Zelda].

(vi) Mia End [more $\lambda w \lambda\left\langle y, w^{\prime}\right\rangle \lambda x x$ has a $\left\langle y, w^{\prime}\right\rangle$-expensive hat] [than Zelda].
}

In step (iii-v), more raises and then a degP is projected with an [deg ${ }^{\circ}$ End] head. In step (vi), Mia raises tracelessly to Spec, degP. [than Zelda] could be merged before or after [deg ${ }^{\circ}$ End]. 
Degrees and segments

Beck (2011) provides a comprehensive overview of these and related issues. In the remainder of this subsection, I want to briefly review Heim's argument and then consider what happens in the current system when modals appear in phrasal comparatives.

This paper began with the familiar idea that tall relates an individual to his height. Various authors have argued instead that tall relates an individual to a set of heights, his actual height and all those below it. As just noted, Heim (2001) provided a particularly compelling argument for this idea which, following Gawron (1995), she refers to as "the monotonicity property of adjective meanings". 12 The argument has to do with necessity modals and one can get a sense of it by considering traffic laws. Such laws generally do not specify a particular speed at which one must travel; instead, a range of speeds is permitted. Nevertheless, we make comparisons in this domain: You have to drive faster on a highway than on a city street. An analysis like in (1)-(2) above will presumably quantify over speeds $d$ that satisfy the open sentence one has to drive $d$-fast on a highway. Without monotonicity, no speed will satisfy this sentence because there is no speed $d$ such that you have to drive that speed on a highway. This problem goes away when gradable predicates associate an individual with a set of degrees, monotonically defined.

Under the segmental analysis described above, Jack can run faster than Jill can be interpreted in terms of the measure function in (78).

$$
\lambda w . \lambda x \cdot \lambda\left\langle y, w^{\prime}\right\rangle . x \operatorname{can}_{w} \text { run }\left\langle y, w^{\prime}\right\rangle \text {-fast. }
$$

That function will assign to Jack in any world $w$, the same degree as (79) below assigns to him in the $w$-accessible world where he runs the fastest, what might be called Jack's 'maximal degree'.

$\lambda w . \lambda x . \lambda\left\langle y, w^{\prime}\right\rangle . x \operatorname{run}_{w}\left\langle y, w^{\prime}\right\rangle$-fast.

In other words, the effect of von Stechow's maximality operator is captured already in the creation of a measure function. Artstein (1998) pointed out that von Stechow's maximality operator actually fails in a situation where there is no 'maximal degree', say if Jack can run at any speed less than $\mathrm{n}$ mph (see also Fox and Hackl 2006 on this point). (78) will assign Jack a degree even in this case. Turning to necessity modals, Jack has to run faster than Jill can be interpreted in terms of the measure function in (80).

$$
\lambda w . \lambda x \cdot \lambda\left\langle y, w^{\prime}\right\rangle . x \text { has-to }_{w} \text { run }\left\langle y, w^{\prime}\right\rangle \text {-fast. }
$$

\footnotetext{
${ }^{12}$ This idea is occasionally made vivid by saying that if Jack is 5 feet tall, then he is also 4 feet tall and he's also 3 feet tall and so on. While this is not a necessary consequence, it does point to another locus of intuitive unease that comes with treating degrees as primitive. See Schwarzschild 2008 for an attempt to make sense of monotonicity in terms of the idea of a threshold.
} 
That function will assign to Jack in any world $w$, the same degree as (79) assigns to him in the $w$-accessible world where he runs the slowest, what might be called Jack's 'minimal degree' and this is as in Heim 2001 . $^{13}$

It seems then that our particular choice for how to derive degrees from primitive grading relations has the benefit that it somehow encodes 'maximality' and 'monotonicity'. Although our measure functions are defined on possible individuals, the logic is the same as in a degree semantics with monotonicity (Rett 2006, 2008: §2; Heim 2006). What should we make of this? If one is persuaded that degrees should not be primitive entities in the semantics, then modals in comparatives represent an argument for the method for deriving them proposed in subsection 3.2. And if one is happy with the intuitions behind that method, then we have an explanation for maximality and monotonicity. They need not be stipulated in the meanings of adjectives and degree heads and they are not detachable - we expect to find these effects whenever degrees are invoked.

\subsection{More on degrees and segments}

In the previous two subsections, we defined degrees in terms of possible individuals, allowing us to handle conjunctions, as Bale (2007) suggested, and then, having done this in a segmental context, we were able to implement his idea about for phrases in a way that avoided the problems pointed out by Schwarz. To conclude, I'd like to briefly discuss two examples that require the tools introduced in section 3 and that make a connection with the discussion in section 2.

(81) below is a variation on the counterfactual example (19) used in subsection 2.2:

(81) If they had hired Holly, she would have made tastier bread than the current chef.

Like (19), the standard PP at the end of (81) is best read de-re. (81) differs from (19) in that the measure function that assigns the current chef a degree in the actual world is denoted by an LF derived predicate. It is that function that assigns to any $x$ and $w$ in its domain, the degree to which $x$ makes tasty bread in $w$. So the -er affix on tastier will need to move to take the whole complement of would in its scope. But once that is done, the semantics is segmental, and so the standard phrase gets to 'use' that function.

In section 2.2, we had de-re readings of counterfactuals and modal statements. I think such readings are possible as well in propositional attitude contexts. See what you think of the following case.

\footnotetext{
${ }^{13} \mathrm{I}$ 'm assuming that $-e r$ scopes over the modal in both of these examples. To be sure, there are readings where the scope if reversed.
} 
Degrees and segments

Suppose the U.S. Internal Revenue Service assessed a tax on us based on the size of our home and suppose they were wildly off, assuming our house to be much larger than it is. Knowing that you are not versant in square footage, I mean to convey the enormity of their error by making reference to the Duke of York, whose home we looked at on a recent vacation in London. I report:

(82) The IRS thinks we own a larger house than the Duke of York.

As far as I know, the IRS has no thought about the Duke of York, or his home, only the actual size of his home is part of the reported thought. The standard phrase is read de-re and the measure function in play is one that assigns to an individual and a world, the size of the home they own in that world.

\section{Summary and Conclusion}

The empirical ground covered in this paper includes: adjective conjunctions under more, the dependency between -er and than, Navajo comparatives with free adjunct standard phrases, degree de-re standard phrases, comparatives containing for phrases and phrasal comparatives with modals. The chief theoretical proposals were reduction of degrees to sets of possible individuals and the idea that Degree constructions involve quantification over directed scale segments. I'd like to end with a few remarks on these two proposals.

A reductionist account of degrees removes mysteries that arise when degrees are primitive: What are degrees? Where do they come from? What determines their ordering? Why does degree quantification into intensional contexts seem uncomplicated as compared to individual quantifying in? What prevents degrees of different kinds from being ordered? Regarding the last question, on the definition of $<$ given in (48), if my weight were ordered below my height, it would follow that any possible individual whose weight was equal to mine would have to be my height or less. In other words, it would be impossible for someone taller than me to have my weight. And the only way my height could be ordered below my weight is if it were impossible for someone heavier than me to have my height. It is these kinds of impossibilities that explain what prevents weights and heights from being ordered. In the future, it may be interesting to consider the development of measurement systems (Díez 1997) and their role in natural language semantics (Sassoon 2010, van Rooij 2011) from the reductionist perspective described here.

When I first read about Navajo comparatives in Bogal-Allbritten 2008, I was puzzled by how much of the meat of comparison could be sandwiched inside an adjunct. I'd become accustomed to thinking of the standard and the comparativemarker as central elements of the comparative. In fact, differentials in English already show more flexibility than I was allowing for. In Hellan 1981, specifier 
differentials ( $5 f t$ taller) are arguments of the comparative morpheme. In Schwarzschild and Wilkinson 2002, it becomes even more important to include differential information in the semantics of the comparative morpheme. And yet, differentials can appear inside adverbial PPs following the rest of the clause:

(83) The bowl is heavier than any of the glasses were, by at least 2 grams.

The segmental framework proposed in section 2 is an attempt to cope with the flexibility languages display in the arrangement of the parts of a comparative construction. I should note an open question for structures considered here. In section 3, -er was assigned the role of introducing segments. What, if anything, performs this task in comparatives without comparative markers, like the Navajo example cited in section 2 ?

The event-like character of the segmental framework leads naturally to the idea that there are thematic roles for segments. Other proposals for extending the notion of thematic role to non-eventive relations include Larson 1991, Abney 1987: 294-5 and Schwarzschild 2006: §6. I'm not sure if ultimately this is the right way to go.

\section{References}

Abney, Steven Paul. 1987. The English noun phrase in its sentential aspect, MIT Phd.

Alrenga, Peter, Christopher Kennedy, and Jason Merchant. 2012. A new standard of comparison. In Nathan Arnett and Ryan Bennett (eds.) 30th West Coast Conference on Formal Linguistics (WCCFL), 32-42. Cascadilla Proceedings Project. Somerville, MA.

Andersen, P. K. 1982. On Universal 22. Journal of Linguistics 18(20). 231-243. http://dx.doi.org/10.1017\%2FS0022226700013591

Artstein, Ron. 1998. Maximality or quantification? A critique of Rullmann (1995) dissertation ch. 2. Rutgers ms.

Bale, Alan Clinton. 2011. Scales and comparison classes. Natural Language Semantics 19(2). 169-190 . doi:10.1007/s11050-010-9068-0.

Bale, Alan. 2007. Boolean and and the semantic correlates of gradable adjectives. Handout for International Conference on Adjectives. Lille (France), 13 - 15 September 2007.

Barker, Chris. 2007. Parasitic scope. Linguistics and Philosophy 30(4). 407-444. http://dx.doi.org/10.1007\%2Fs10988-007-9021-y

Beck, Sigrid. 2011. Comparison constructions. In Claudia Maienborn, Klaus von Heusinger and Paul Portner (eds.), Semantics: An International Handbook of Natural Language Meaning. 1341-1390. Mouton de Gruyter. Berlin, 
Degrees and segments

Beck, Sigrid. 2012. Quantifiers in than-clauses. Semantics and Pragmatics 3(1). 1-72. http://dx.doi.org/10.3765/sp.3.1

Bhatt, Rajesh and Roumyana Pancheva. 2004. Late merger of degree clauses. Linguistic Inquiry 35(1). 1-45 http://dx.doi.org/10.1162\%2F002438904322793338

Bhatt, Rajesh and Shoichi Takahashi. 2011. Reduced and unreduced phrasal comparatives. Natural Language and Linguistic Theory 29(3). 581-620. http://dx.doi.org/10.1007/s11049-011-9137-1

Bobaljik, Jonathan David. 2012. Universals in Comparative Morphology: Suppletion, Superlatives, and the Structure of Words. MIT Press

Boeckx, Cedric and Norbert Hornstein. 2004. Movement under control. Linguistic Inquiry 35(3). 431-452. http://dx.doi.org/10.1162\%2F0024389041402625

Bogal-Allbritten, Elizabeth. 2008. Gradability and degree constructions in Navajo. B.A. thesis, Swarthmore College.

Bogal-Allbritten, Elizabeth. 2013. Decomposing notions of adjectival transitivity in Navajo. Natural Language Semantics doi:10.1007/s11050-012-9093-2

Cooper, Robin. 1978. Variable binding and relative clauses. In Franz Guenthner and Siegfried J. Schmidt (eds.) Formal Semantics and Pragmatics for Natural Languages, 131-169. Reidel. Dordrecht.

Cresswell, M. J. 1976. The semantics of degree. In Barbara H. Partee (ed.), Montague Grammar, 261-292. Academic Press.

Cresswell, M. J. 1984. Comments on von Stechow. Journal of Semantics 3(1). 7981. http://dx.doi.org/10.1093\%2Fjos\%2F3.1-2.79

Cresswell, Maxwell J. and Arnim von Stechow. 1982. De re belief generalized. Linguistics and Philosophy 5(4). 503-535. http://dx.doi.org/10.1007\%2FBF00355585

Díez, José A. 1997. A hundred years of numbers. An historical introduction to measurement theory 1887-1990; Part I: The formation period. Two lines of research: Axiomatics and real morphisms, scales and invariance," Studies in History and Philosophy of Science Part A. 28(1). 167-185 http://dx.doi.org/10.1016\%2FS0039-3681\%2896\%2900014-3

Dotlačil, Jakub. 2010. Anaphora and distributivity. A study of same, different, reciprocals and others. UIL-OTS, Utrecht University PhD.

Faller, Martina. 2000. Dimensional adjectives and measure phrases in vector space semantics. In Martina Faller, Stefan Kaufmann and Marc Pauly (eds.), Formalizing the Dynamics of Information, 151-170. CSLI Publications, Stanford.

Fox, Danny and Martin Hackl. 2006. The universal density of measurement. Linguistics and Philosophy 29(5). 537-586. doi:10.1007/s10988-006-9004-4. Gajewski, Jon Robert. 2005. Neg-raising: polarity and presupposition. MIT PhD. 
Gawron, Jean Mark. 1995. Comparatives, superlatives and resolution. Linguistics and Philosophy 18(4). 333-380. http://dx.doi.org/10.1007/BF00984929

Grosu, Alexander and Julia Horvath. 2006. Reply to Bhatt and Pancheva's 'Late Merger of Degree Clauses': The irrelevance of (non)conservativity. Linguistic Inquiry 37(3). 457-483 http://dx.doi.org/10.1162\%2Fling.2006.37.3.457

Heim, Irene. 1985. Notes on comparatives and related matters. University of Texas, Austin, ms.

Heim, Irene. 1990. Phrasal comparatives and ellipsis. MIT ms.

Heim, Irene. 2001. Degree operators and scope. In Caroline Féry \& Wolfgang Sternefeld (eds.), Audiatur vox sapientiae: A festschrift for Arnim von Stechow, 214-239. Berlin: Akademie Verlag.

Heim, Irene. 2006. Little. In Masayuki Gibson and Jonathan Howell (eds.), Semantics and Linguistic Theory 16 (SALT), 35-58. Ithaca, NY: Cornell University. doi:1813/7579.

Hellan, Lars. 1981. Towards an Integrated Analysis of Comparatives (Ergebnisse und Methoden moderner Sprachwissenschaft 11). Tübingen: Narr.

Hoeksema, Jack. 1983. Negative polarity and the comparative. Natural Language and Linguistic Theory 1(3). 403-434. doi:10.1007/BF00142472.

Kennedy, Christopher. 1997. Projecting the adjective: The syntax and semantics of gradability and comparison. University of California, Santa Cruz, $\mathrm{PhD}$.

Kennedy, Christopher. 2007. Vagueness and grammar: The semantics of relative and absolute gradable adjectives. Linguistics and Philosophy 30(1). 1-45. http://dx.doi.org/10.1007/s10988-006-9008-0

Keshet, Ezra. 2010. Situation economy. Natural Language Semantics 18(4). $385-$ 434 http://dx.doi.org/10.1007\%2Fs11050-010-9059-1

Klein, Ewan. 1991. Comparatives. In Arnim von Stechow and Dieter Wunderlich (eds.), Semantik/Semantics: An International Handbook of Contemporary Research. 673-691. Walter de Gruyter.

Kratzer, Angelika. 1996. Severing the external argument from its verb. In Johan Rooryck and Laurie Zaring (eds.), Phrase Structure and the Lexicon. 109-37. Kluwer, Dordrecht.

Kusumoto, Kiyomi. 2005. On the quantification over times in natural language. Natural Language Semantics 13(4). 317-357. http://dx.doi.org/10.1007\%2Fs11050-005-4537-6

Larson, Richard K. 1991. The projection of DP and DegP. State University of New York at Stony Brook ms.

Lechner, Winifried. 2008. On binding scope and ellipsis scope. In Kyle Johnson (ed.) Topics in Ellipsis, 154-182, 273-276 Cambridge University Press. 
Degrees and segments

Malamud, Sophia A. 2012. The meaning of plural definites: A decision-theoretic approach. Semantics and Pragmatics 5(3). 1-58.

http://dx.doi.org/10.3765/sp.5.3

Matushansky, Ora. 2013. More or better: On the derivation of synthetic comparatives and superlatives in English. To appear in Ora Matushansky and Alec Marantz,(eds.), Distributed Morphology Today: Morphemes for Morris Halle. Cambridge, MA: MIT Press

McConnell-Ginet, Sally. 1973. Comparative constructions in English: A syntactic and semantic analysis. University of Rochester, $\mathrm{PhD}$.

Musan, Renate. 1999. Temporal interpretation of noun phrases. Linguistics and Philosophy 22(6). 621-661.

Napoli, Donna Jo. 1983. Comparative ellipsis: a phrase structure analysis. Linguistic Inquiry 14. 675-694.

Nissenbaum, Jon and Bernhard Schwarz. 2008. Two puzzles about infinitivals with too. In Natasha Abner and Jason Bishop (eds.), 27th West Coast Conference on Formal Linguistics (WCCFL). 344-352. Somerville, MA: Cascadilla Proceedings Project.

Rett, Jessica. 2006. How many maximizes in the Balkan sprachbund. In Masayuki Gibson and Jonathan Howell (eds.), Semantics and Linguistic Theory 16 (SALT). 190-207. Ithaca, NY: Cornell University. doi:1813/7588.

Rett, Jessica. 2008. Degree modification in natural language. Rutgers PhD. van Rooij, Robert. 2011. Measurement and interadjective comparisons. Journal of Semantics 28(3). 335-358 doi:10.1093/jos/ffq018

Rullmann, Hotze. 1995. Maximality in the Semantics of Wh-Constructions. Ph.D. thesis, University of Massachusetts at Amherst. URL http://scholarworks.umass.edu/dissertations/AAI9524743/

Sassoon, Weidman Galit. 2010. Measurement theory in linguistics. Synthese 174(1). 151-180 http://dx.doi.org/10.1007\%2Fs11229-009-9687-5

Schauber, Ellen. 1979. The syntax and semantics of questions in Navajo. MIT $\mathrm{PhD}$.

Schwager, Magdalena. 2011. Speaking of qualities. In Ed Cormany, Satoshi Ito and David Lutz (eds.) Semantics and Linguistic Theory 19 (SALT). 395-412. eLanguage CLC Publications,

Schwarz, Bernhard. 2010. A note on for-phrases and derived scales. Handout for talk at Sinn und Bedeutung 15, September 9-11, 2010.

Schwarzschild, Roger and Karina Wilkinson. 2002. Quantifiers in comparatives: A semantics of degree based on intervals. Natural Language Semantics 10(1). 1-41. doi:10.1023/A:1015545424775.

Schwarzschild, Roger. 2006. The role of dimensions in the syntax of noun phrases. Syntax 9(1). 67-110 http://dx.doi.org/10.1111\%2Fj.14679612.2006.00083.x 
Schwarzschild, Roger. 2008. The semantics of comparatives and other degree constructions. Language and Linguistics Compass 2(2). 308-331. doi:10.1111/j.1749-818X.2007.00049.x.

Sharvit, Yael and Penka Stateva 2002. Superlative expressions, context, and focus. Linguistics and Philosophy 25(4). 453-504. doi:10.1023/A:102087580979

Stassen, Leon. 1985. Comparison and Universal Grammar. Oxford: Basil Blackwell.

von Stechow, Arnim. 1984. Comparing semantic theories of comparison. Journal of Semantics 3(1-2). 1-77. doi:10.1093/jos/3.1-2.1.

Szabolcsi, Anna and Bill Haddican. 2004. Conjunction meets negation: A study of cross-linguistic variation. Journal of Semantics 21(3). 219-250. http://dx.doi.org/10.1093/jos/21.3.219.

Tonhauser, Judith. 2007a. Nominal tense? The meaning of Guaraní nominal temporal markers. Language 83(4). 831-869 http://dx.doi.org/10.1353\%2Flan.2008.0037

Tonhauser, Judith. 2007b. The temporal semantics of noun phrases: Evidence from Guarani (Paraguay). Stanford $\mathrm{PhD}$

Winter, Yoad. 2005. Cross-categorial restrictions on measure phrase modification. Linguistics and Philosophy 28. 233-267. http://dx.doi.org/10.1007\%2Fs 10988-004-1469-4

Young, Robert W. and William Morgan. 1987. The Navajo Language: A Grammar and Colloquial Dictionary. Albuquerque: University of New Mexico Press.

Roger Schwarzschild

18 Seminary Place

New Brunswick, NJ 08901

rogerschwarzschild@gmail.com 